\title{
Design of control system using electric cylinder for automated measurement of bearing shell crush height
}

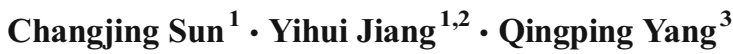

Received: 22 May 2017 / Accepted: 13 October 2017 / Published online: 7 November 2017

(C) The Author(s) 2017. This article is an open access publication

\begin{abstract}
This paper presents the design of a fast and accurate control system using electric cylinder for the automated measurement of bearing shell crush height, which is an important parameter for quality control and needs to be measured in a particular test loading according to ISO3548-3:2012 standard. A test bench has been developed and an electric cylinder is used to provide the required test loading pressure with the LabVIEW platform. In order to meet the demands for high accuracy, fast speed, and stability, the control method of electric cylinder has been designed for industrial applications based on PLC. The control methods include automatic origin seeking and reset when powering up, fast and accurate pressure loading, generating control parameters by self-learning, and returning to the origin and pressure calibration. The fast and accurate pressure loading process consists of position preloading, pressure preloading, pressure coarse loading, and pressure fine loading. The experimental results have shown that the election cylinder can meet the application requirements, the control methods for pressure loading are accurate and fast, and the control system is stable and reliable. They will have potentials for wide industrial applications.
\end{abstract}

Changjing Sun

scj@cjlu.edu.cn

Qingping Yang

qingping.yang@brunel.ac.uk

1 College of Quality and Safety Engineering, China Jiliang University, Hangzhou 310018, China

2 Holley Technology Co. Ltd., Hangzhou 310023, China

3 College of Engineering, Design and Physical Sciences, Brunel University London, Uxbridge UB8 3PH, UK
Keywords Electric cylinder · Bearing shell crush height . PLC $\cdot$ Pressure pulsation

\section{Introduction}

Bearing shell is a critical part of engine and is widely used in automobile industry. The crush height of a bearing shell is defined as the value by which a half bearing, fitted in a checking block of bore diameter, under a predetermined checking load, exceeds the defined peripheral length of the checking block bore (as shown in Fig. 1) [1]. The crush height is a critical parameter indicating the quality of the bearing shell. At present, manual and automatic methods are the two main types of measuring crush height, in which the checking load is applied respectively by torque wrench, oil cylinder [2], or gas cylinder [3]. The use of manual method is limited because of its obvious shortcoming. The automated measurement of bearing shell crush height is crucial for the online quality control and thus has great significance. Since the cross section of a bearing shell in its natural state is not a standard semicircle, it needs to be fitted into a checking block with a predetermined checking load [1]. The fast and accurate control of the loading pressure is the key to the automated measurement. Generally, the pressure pulsation of hydraulic system often exceeds $5 \%$ in the nominal pressure of 2 to $12.5 \mathrm{MPa}$ $[4,5]$. And the pressure pulsation of pneumatic system still exceeds $8 \%$ even after the complicated stabilization of pneumatic source [3]. Using hydraulic and pneumatic systems to provide loading pressure for bearing height measurement thus takes a long time to wait for pressure pulsation to meet the standard requirements due to their intrinsic characteristics. Besides, possible leakage and environmental pollution of hydraulic and pneumatic cylinders also restrict their applications. This paper has proposed the use of electric cylinder for 


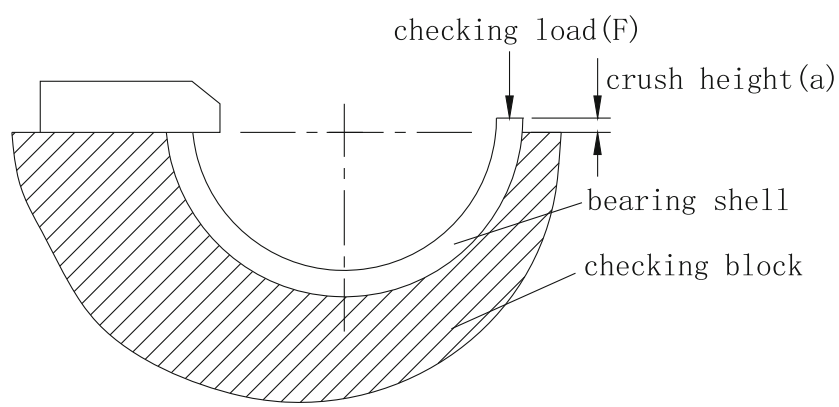

Fig. 1 Measuring principle of bearing shell crush height

applying pressure load in the automated system for bearing shell crush height measurement.

Electric cylinder is the integration of a servo motor and a lead screw. The rotary motion of the servo motor is converted into a linear translation of the electric cylinder rod by means of the lead screw, thus with the advantages of a servo motor inherited in the electric cylinder $[6,7]$. The speed, position, and thrust of an electric cylinder can be accurately controlled by setting and controlling appropriately the rotary speed, position, and moment of the servo motor.

Compared with the traditional pneumatic and hydraulic systems, the power of an electric cylinder comes from the servo motor which does not need oil, air, or other media. It thus has high quality and stable performances and is less susceptible to environmental and other factors. Owing to the closed-loop control of the servo motor, the electric cylinder has capability to produce pressure output with better precision than other power systems [8]. The main disadvantage of an electric cylinder is its relatively high cost.

With the development of modern industry, electric cylinders are used in more and more industrial equipment, which also calls for more and stricter operating requirements for electric cylinders. These for example include some special requirements in the emerging applications, such as using electric cylinders to provide pressure to fix the measured object in metrology industry.

For the measurement of bearing shell crush height, the bearing shell needs to be pressed in the checking block with a specified test load. ISO3548-3: 2012 has given relevant stability requirements for the test load (see Table 1) [1]. If an electric cylinder is used to provide the test load, the electric

Table 1 Load pressure stability requirements

\begin{tabular}{lll}
\hline$F(\mathrm{~N})$ & & $F$ deviation $( \pm \%)$ \\
\hline From & To & \\
\hline- & 2000 & 1.25 \\
2000 & 5000 & 1.0 \\
5000 & 10,000 & 0.75 \\
10,000 & 50,000 & 0.5 \\
50,000 & - & 0.25 \\
\hline
\end{tabular}

cylinder pressure pulsation characteristics must be first understood to ensure that it meets the standard requirements and the control method for the high precision and fast pressure loading by the electric cylinder can then be designed; both of these are presented and discussed in this paper.

\section{Measurement of pressure pulsation characteristics of electric cylinder}

The measurement of pressure pulsation of electric cylinder is performed in the test bench shown in Fig. 2. The electric cylinder is connected with the stand, and the pressure sensor is connected with the electric cylinder rod through the screw. The pressure block is fixed at the other end of the pressure sensor. The rear limit position sensor, the origin sensor, the origin proximity sensor, and the front limit position sensor are all mounted on the outside of the electric cylinder and are sequentially arranged in the order from the rear of the electric cylinder to the front. The distance between the origin proximity sensor and the origin sensor is 2 to $5 \mathrm{~mm}$. The cylinder movement and pressure loading are driven by the PC-based PLC control software, while a NI-DAQ data acquisition card and LabVIEW programs are used to collect and store the pressure data [9].

Transcell BSS-1.5T pressure sensor is selected in this test, which has a full-scale nonlinearity of $\pm 0.03 \%$, a repeatability of $\pm 0.03 \%$, and a safety load of $150 \%$ with the output signal $(0-10 \mathrm{~V})$ representing the test pressure. The NI USB-6210 data acquisition card (16-bit resolution) is used to acquire the signals. The main parameters of the electric cylinder used in the test are the following: a Panasonic A5 servo motor is connected with a lead screw of $5-\mathrm{mm}$ pitch and $60-\mathrm{mm}$ stroke through $1 / 2$ reduction ratio. The servo motor drive is controlled by dedicated software.

According to the low-frequency characteristics of the mechanical vibration, the sampling frequency is first set to

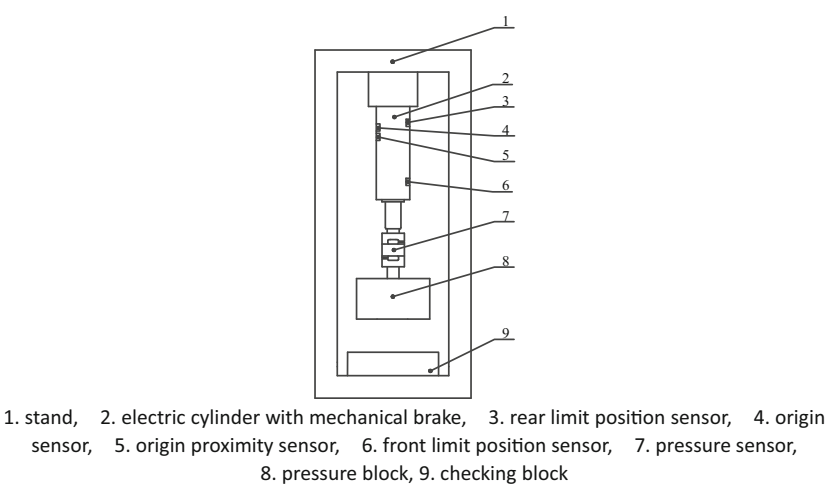

Fig. 2 Test bench of control system for electric cylinder. (1) Stand, (2) electric cylinder with mechanical brake, (3) rear limit position sensor, (4) origin sensor, (5) origin proximity sensor, (6) front limit position sensor, (7) pressure sensor, (8) pressure block, and (9) checking block 
$10 \mathrm{kHz}$ with a low-pass filtering is needed. The main analysis is performed of the pressure signals generated after the electric cylinder rod becomes compressed and has stopped for a while. The signals corresponding to this time interval are extracted with Hanning window and FFT to analyze the main frequencies in frequency domain. The main frequencies of the electric cylinder pressure pulsation can then be obtained, and the pressure fluctuation at this frequency can also be calculated [10].

The raw pressure signals can be filtered with a low-pass filter with a cutoff frequency of $500 \mathrm{~Hz}$, and its power spectrum is then analyzed, as shown in Fig. 3.

Through pressure power spectrum analysis in repeated experiments, it can be seen that the most obvious frequency band is below $10 \mathrm{~Hz}$. The frequency range with significant pressure fluctuation is between 0 and $200 \mathrm{~Hz}$, which is consistent with the characteristics of low mechanical vibration frequency.

The load tolerance of electric cylinder output pressure pulsation can be reflected by the range of the data. Based on the above analysis results, the signal is low-pass filtered again with a cutoff frequency of $200 \mathrm{~Hz}$. A section of signal is acquired when the electric cylinder loading and its output are both stable, and its time domain signal can be seen in Fig. 4.

In the crush height measurement, the time for reading the displacement data is designed to be within $0.5 \mathrm{~s}$. Therefore, when calculating the percentage of load tolerance, the pressure data of $0.5 \mathrm{~s}$ duration is obtained for each group each time. The average value $U$ is taken as the standard pressure value, the range $\delta$ as the pressure pulsation value under the experimental conditions. According to the linear characteristics of the selected pressure sensor, the pressure $L$ has a linear relationship with the output voltage $U$ [11]. The output voltage range is 0 to $10 \mathrm{~V}$; the output is $0 \mathrm{~V}$ at no-load and $10 \mathrm{~V}$ at a full load of $1500 \mathrm{~kg}$.

The fitted pressure-voltage function is the following:

$L=150 U$.

The pressure pulsation and the load tolerance are calculated for the pressure data of three different time intervals under the

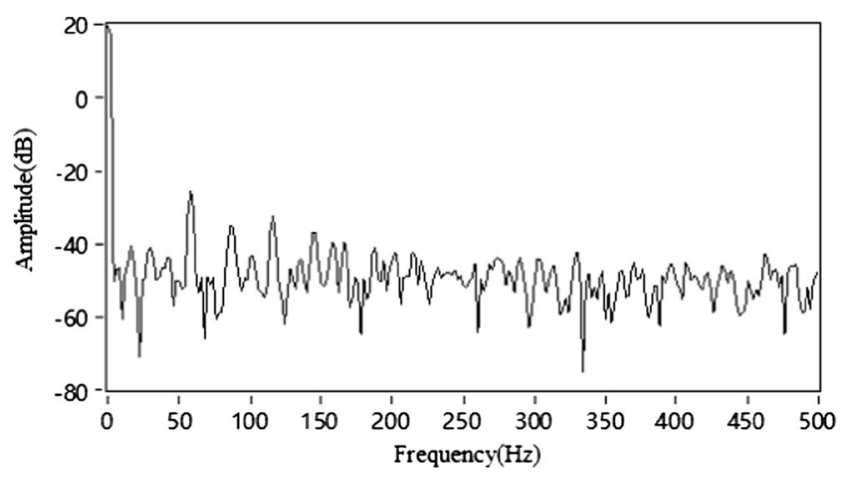

Fig. 3 Power spectrum analysis of pressure pulsation

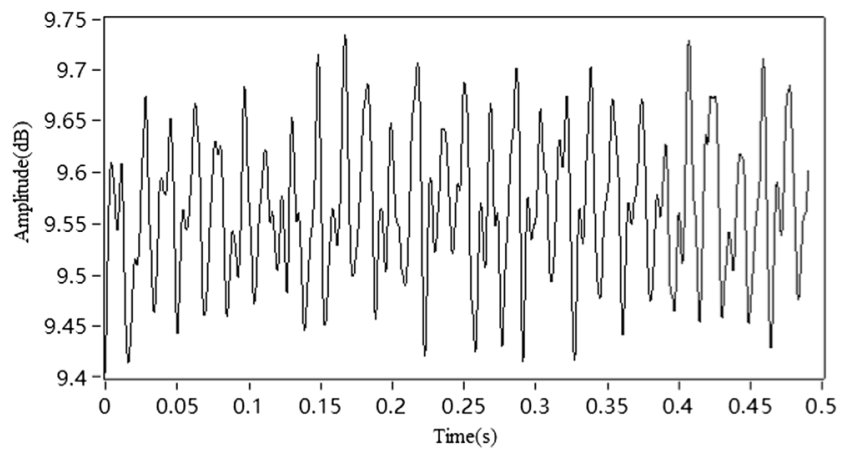

Fig. 4 Time domain analysis of pressure pulsation

same pressure. The results of the three calculations are shown in Table 2.

Compared with Table 1, the output pressure pulsation of the electric cylinder is less than the standard requirement of $\pm 0.75 \%$. The output voltage pulsation of the electric cylinder is therefore within the acceptable range, in line with application requirements. Multiple experiments and calculations are similarly carried out at different load pressures; the pressure pulsations all satisfy the standard requirements.

\section{High precision and rapid loading control method}

Having verified that the output pressure of the electric cylinder meets the application requirements, the control method for the electric cylinder pressure needs to be designed $[12,13]$. The measurement process requires a large range of pressure output, high control accuracy, fast response with the shortest settling time, and no collision during the loading process. Therefore, the control method for the loading system is very important, and it should meet the following requirements: (1) fast loading speed, (2) high precision, (3) flexible loading, and (4) suitable for different pressure target values.

The overall control flow of the pressure loading is shown in Fig. 5. If the electric cylinder is not stopped at the origin at the starting point, the origin seeking and reset are performed. Before the pressure loading, it is necessary to decide according to the actual situation whether or not for the system to selflearn to generate the control parameters. The pressure loading consists of four stages: position preloading, pressure preloading, pressure coarse loading, and pressure fine loading. Finally, the electric cylinder returns to the origin and calibrates the pressure to prepare for the next loading.

Table 2 Experimental results for load tolerances

\begin{tabular}{lll}
\hline Load pressure $L(\mathrm{~kg})$ & Pulsation range $\delta(\mathrm{kg})$ & Load tolerance $( \pm \%)$ \\
\hline 955.5 & 3.31 & 0.17 \\
956.4 & 3.20 & 0.17 \\
955.4 & 3.50 & 0.18 \\
\hline
\end{tabular}




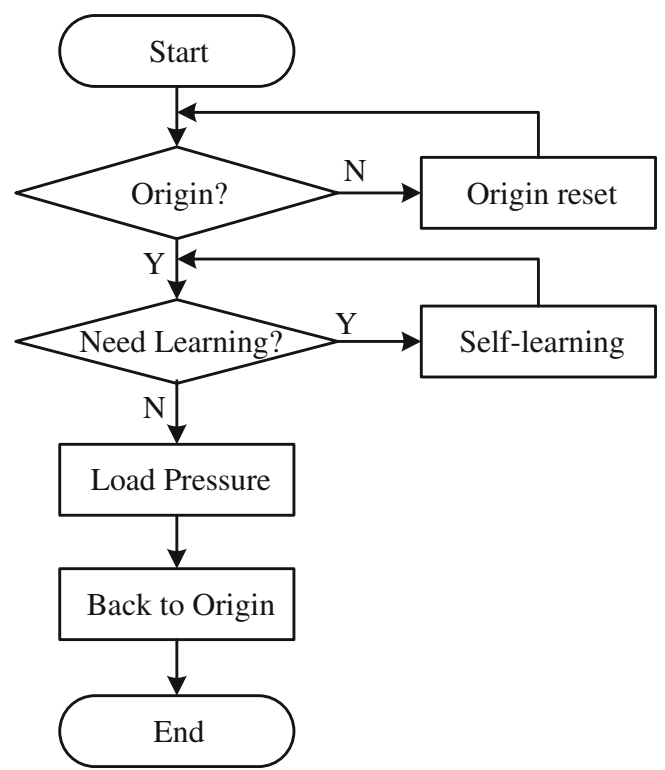

Fig. 5 Flow chart of the control method of electric cylinder

A common general PLC is selected as a control center based on the consideration of the industrial environment. The basic structure of the closed-loop control for electric cylinder is shown in Fig. 6. The PLC control algorithm controls the electric cylinder through driving the servo motor. The output pressure of the electric cylinder is fed back to the PLC via a pressure sensor, forming a closed-loop control system. In the executing controller, the servo motor driver and the servo motor also form a closed-loop system. The good characteristics of the servo motor can ensure the good quality of the controller.

Due to the refreshing time of the PLC and AD conversion module, it is necessary to consider and overcome the hysteresis of pressure signal feedback. The magnetic switch and other switch signals can overcome the refreshing time through the PLC's rapid response port or system interrupt mode. For pressure signals, the pressure loading speed can be slowed down to reduce the impacts of signal hysteresis.

The design of fast high precision loading control method for the electric cylinder pressure includes four parts: the control method for the automated origin position seeking and reset, the fast and accurate pressure loading control method, the self-learning control parameter method, and the control method for accurate return to the origin position and calibration of the pressure.
1) Control method for the automated origin position seeking and reset

The servo motor receives the PLC signal in the form of "pulse + direction" and runs in the position control mode. The servo motor is set to receive 1500 pulses per turn.

When starting, the origin sensor status is read. If the origin sensor has no signal, i.e., the electric cylinder is not stopped at the origin, it begins to seek the origin position. The PLC first drives the servo cylinder to quickly retract and reads the signals of the four sensors on the cylinder body: if the rear limit sensor signal is detected during the reverse process, the direction of rotation of the servo motor is switched and the motor cylinder rod extends out quickly; if the front limit sensor signal is detected in the forward moving direction, the direction of rotation of the servo motor is switched, the electric cylinder rod is quickly driven back; if the origin proximity sensor signal is detected in the retraction process, the servo motor immediately slows down, and the electric cylinder rod continues to slowly (less than $2 \mathrm{~mm} / \mathrm{s}$ ) retreat until the detection of the origin sensor signal, the servo motor stops rotation, completing the origin position seeking and reset.

\section{2) Fast and accurate pressure loading control method}

In order to achieve fast and accurate pressure loading with smooth loading and no collision, the fast and accurate loading control method is studied and developed. The pressure loading process begins when the pressure block contacts with the checking block (shown in Fig. 2). Experiment was designed to obtain the relationship between the pressure and the pulse during the loading process.

The experimental process is designed as follows: when the pressure block is about to contact the checking block, instructions are executed to send pulses to the electric cylinder, with the pressure recorded every 50 pulses. Three sets of experimental data are used to determine the relationship between the pulse and pressure (see Fig. 7). It can be seen that when the pressure block contacts with the checking block, the pressure shows a sudden change point and the pressure loading begins. The relationship between the number of pulses and the pressure basically has a linear correlation. The pressure loading control method can be derived from this.

The fast and accurate pressure loading from the origin to the complete loading is divided into four stages: position preloading,
Fig. 6 Basic structure of the closed-loop control for electric cylinder

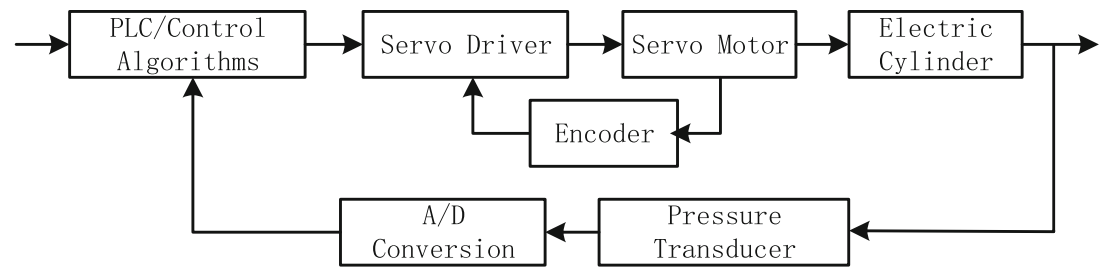




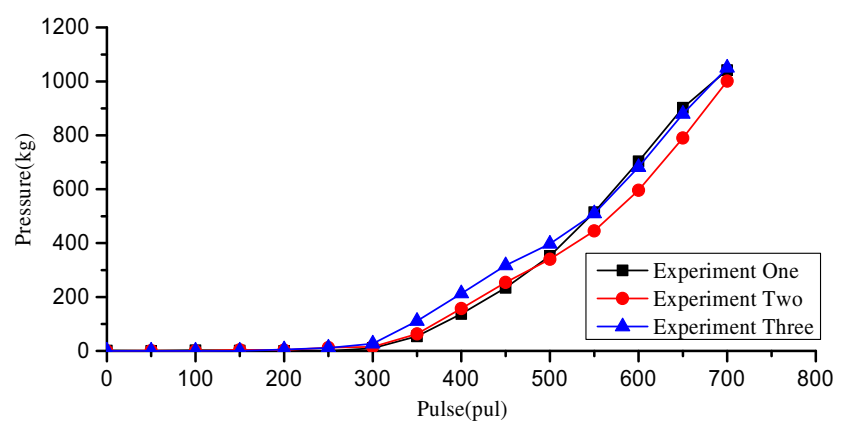

Fig. 7 Pulse-pressure experimental results

pressure preloading, pressure coarse loading, and pressure fine loading. The sequence chart of pulse, velocity and displacement of the cylinder rod, and pressure are shown in Fig. 8.

Position preloading

From the origin, the electric cylinder rod is quickly accelerated to the maximum speed (up to $200 \mathrm{~mm} / \mathrm{s}$ for the selected electric cylinder), and it moves forward for a certain distance and then begins to slow down until the pressure block is $0.5 \sim 2 \mathrm{~mm}$ from the checking block to ensure that there is no collision (at $t_{3}$ in Fig. 8). During this stage, the electric cylinder rod has moved the longest distance with the fastest speed. The distance traveled by the electric cylinder rod is calculated from the total number of pulses recorded during the self-learning (detailed below), and the acceleration and deceleration points are automatically calculated after the acceleration is set.

Pressure preloading

After the position preloading is completed, the motor cylinder drives the pressure block to move forward at a speed of 0.3 to $0.6 \mathrm{~mm} / \mathrm{s}$; the pressure is monitored in real time through the pressure sensor until the pressure changes abruptly when it stops immediately (at $t_{4}$ in Fig. 8 ). At this moment, the pressure block and the checking block have just made contact; the pressure is very small.

Pressure coarse loading

The pressure-pulse function obtained by the experiment is preloaded into the control system. Eighty to ninety-five percent of the required pressure is taken as the target pressure, and the corresponding number of pulses is calculated. The electric cylinder drives the pressure block at $0.6-1 \mathrm{~mm} / \mathrm{s}$ loading speed to execute the above number of pulses (at $t_{5}$ in Fig. 8).

Pressure fine loading

The electric cylinder continues to drive the pressure block to move forward, obtains the difference between the current pressure and the required pressure in real time and decelerates at different accelerations according to the magnitude of the difference. If the pressure difference is between 80 and $160 \mathrm{~kg}$, the servo motor is driven at a speed of 200 to 300 pulses per second. If the pressure difference is between 40 and $80 \mathrm{~kg}$, the speed is 100 to 200 pulses per second. If the pressure difference is less than $40 \mathrm{~kg}$, the servo motor is driven at a speed of 10 to 50 pulses per second. The closer the current

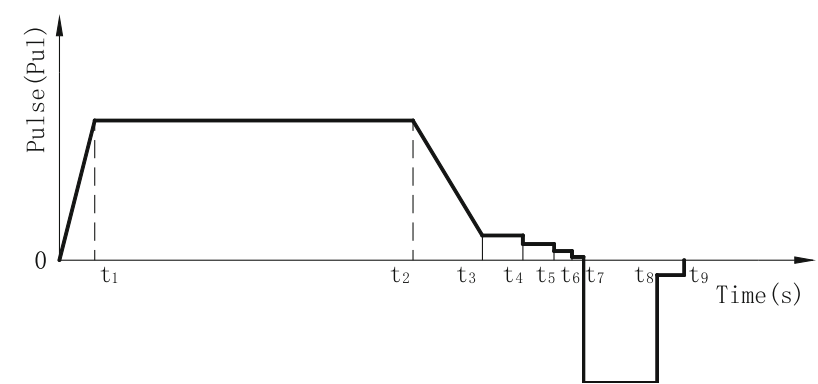

(a) Sequence chart of pulse driving the servo motor

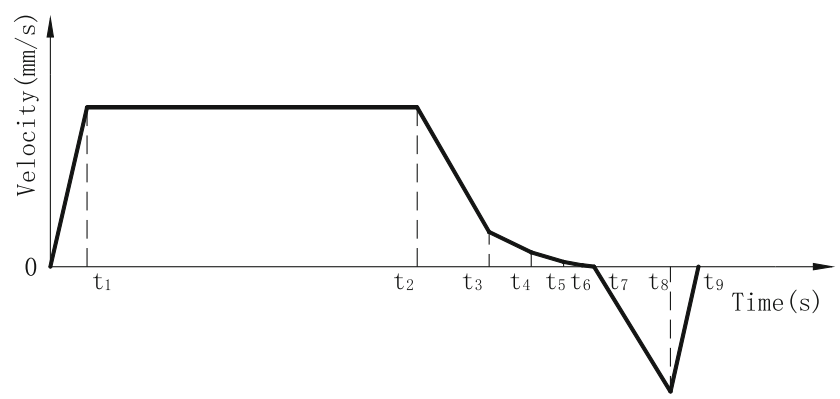

(b) Sequence chart of the velocity of electric cylinder rod



(c) Sequence chart of the distance of the checking block

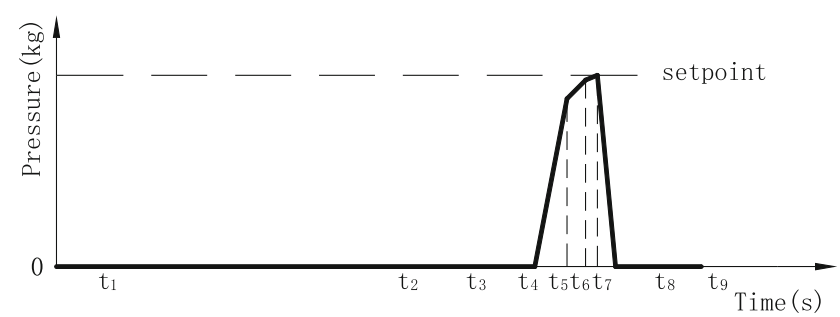

(d) Sequence chart of pressurel oad

Fig. 8 Sequence charts of several parameters of electric cylinder. a Sequence chart of pulse driving the servo motor. b Sequence chart of the velocity of electric cylinder rod. c Sequence chart of the distance of the checking block. d Sequence chart of pressure load

pressure is to the required pressure, the slower the loading rate. The effect of the hysteresis of the pressure feedback loop is reduced without reducing the overall loading efficiency until the real-time pressure reaches the set point; the loading stops with mechanical braking to complete the pressure loading (at $t_{7}$ in Fig. 8)

3) Method for self-learning to generate control parameters

When bearing shells with different crush heights are pressed into the checking block, the protruding lengths above 
the checking block bore reference are different (see Fig. 1). As mentioned earlier, in the position preloading stage, the electric cylinder rod should move at the fastest speed to improve the efficiency of the measurement, but no collision should happen. Therefore, the stop position of the electric cylinder rod in the position preloading stage also needs to be adjusted. This paper proposed the self-learning method based on the relaxed operation with the external input data to improve the efficiency of measurement and generate the required electric cylinder control parameters automatically according to different applications and pressure settings.

Relaxed operation

Relaxed operation runs with the same four stages as the fast and precise pressure loading, but the control strategy is the accuracy taking precedence over the speed. The control method differs in the following aspects: 1) it stops earlier during the position pre-loading stage; 2 ) the slower speed loading during the pressure preloading; and 3) when the coarse loading begins, the total number of pulses from the origin to the current position received from the PLC are read and stored for use at normal run-time during the position preloading phase. The relaxed operation will allow the required electric cylinder control parameters to be automatically stored (i.e., self-learnt).

External input data

In some cases where human intervention is required to adjust the operating state, the total number of pulses stored in the relaxed operation control phase may be compensated with the external input pulse compensation value to meet the control requirements. The self-learning process is combined with human intervention to achieve higher loading efficiency.

4) Control method for accurate return to the origin and pressure calibration

Accurate return to the origin

After the completion of the pressure loading and the subsequent tasks, the electric cylinder moves towards the origin at the maximum speed. When the reading shows the origin is close by, it quickly slows down to slow motion and continues its operation in the system interruption mode until the original sensor signal is received when it stops and the return to the origin is completed. This process is similar to the starting process to seek the origin, but is more efficient.

Pressure calibration

Since the output value of the pressure sensor at no-load is not necessarily 0 , and because of mechanical reasons, when the electric cylinder stays at the origin, the pressure is not necessarily in no-load state. Therefore, after each reset of the electric cylinder, the output pressure value of the pressure sensor at no-load requires processing. The specific method is when the electric cylinder returns to the origin, the pressure sensor output is read and set it to 0 , ready for the next pressure
Table 3 Repeated measurement data for one bearing shell

\begin{tabular}{llllll}
\hline No. & 1 & 2 & 3 & 4 & 5 \\
Pressure & 390.75 & 391.50 & 391.50 & 391.50 & 391.00 \\
No. & 6 & 7 & 8 & 9 & 10 \\
Pressure & 392.50 & 390.75 & 391.00 & 390.50 & 390.50 \\
No. & 11 & 12 & 13 & 14 & 15 \\
Pressure & 391.00 & 391.75 & 391.25 & 391.75 & 392.75 \\
No. & 16 & 17 & 18 & 19 & 20 \\
Pressure & 392.00 & 392.25 & 392.75 & 392.75 & 393.50 \\
No. & 21 & 22 & 23 & 24 & 25 \\
Pressure & 394.00 & 393.75 & 393.50 & 392.75 & 393.75 \\
No. & 26 & 27 & 28 & 29 & 30 \\
Pressure & 392.00 & 392.50 & 393.50 & 390.75 & 390.50 \\
\hline
\end{tabular}

loading. The use of this method requires that the pressure sensor has normal function with good linearity [14].

\section{Pressure loading experiments}

The above control method described in the previous section is run to carry out the pressure loading experiment. The experimental design is as follows: the target pressure value is set to $390 \mathrm{~kg}$, the pressure loading process is run 30 times continuously, and the pressure data is recorded to analyze its stability (see Table 3 and Fig. 9).

It can be seen that the pressure range is $3.5 \mathrm{~kg}$, i.e., the pressure fluctuation is $3.5 \quad 392.01= \pm 0.45 \%$, with good pressure data stability.

\section{Conclusions}

Use of electric cylinder to provide test load offers good pressure stability, satisfying the application standards. The experiments have shown that the pressure control system of electric cylinder based on PLC is stable and reliable. The actual

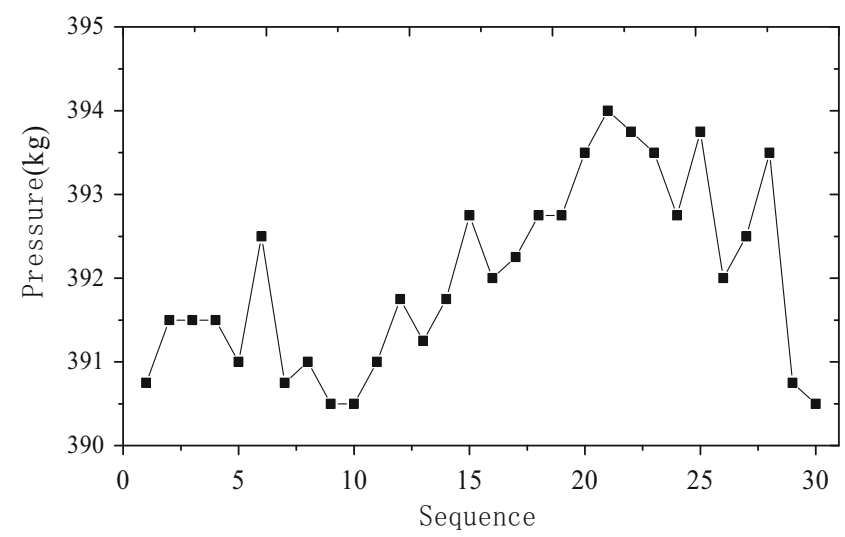

Fig. 9 Pressure curve 
pressure data is slightly higher than the set value, but the error is within the acceptable range. Its repeatability also meets the requirements.

Compared with the existing pneumatic and hydraulic systems, the high precision and fast loading control method of electric cylinder pressure presented in this paper has advantages of adjustable starting point (origin), quick reset, fast, accurate, and stable pressure loading, and environmentfriendliness. With this method, the optimal control parameters can be generated for different applications through self-learning, and it has wide application prospects.

Open Access This article is distributed under the terms of the Creative Commons Attribution 4.0 International License (http:// creativecommons.org/licenses/by/4.0/), which permits unrestricted use, distribution, and reproduction in any medium, provided you give appropriate credit to the original author(s) and the source, provide a link to the Creative Commons license, and indicate if changes were made.

\section{References}

1. British Standard Institution, BSI ISO 3548-3. Plain bearings Thin-walled half bearings with or without flange, Part 3: Measurement of peripheral length. London: British Standards Institute, 2012

2. Lin XJ. Design and development of double side loading thin wall bearing girth detection system. Internal Combustion Engines and Parts. 2007(2):8-12
3. Xie SX, Deng HT, Zhang XF (1982) Way of solving the question of air supply stabilization in pneumatic measuring instrument for measuring half peripheral length of bearing shell. Internal Combustion Engine Parts. 04:31-33

4. Liu LG (2001) Pressure pulsation measurement of axial piston pump. Shandong Coal Technol 02:34-35

5. He ZY, He QH, He SH (2010) Experimental study of pressure pulsation in the loop of pumping source of hydraulic system. Mining Machinery 38(20):35-38

6. Zhao M. The research of the electric power steering system developing platform. Chongqing Jiaotong University, 2012

7. Zhang HY, Hu M Technology and application of spot welding head on robot. Welding Technol 38:44-46

8. Wei YH, Han LJ, Xu C (2014) Design of electric cylinder servo control system using PLC. Electrical Transmission 44(10):60-63

9. Yan Y, Ma ZQ, Yang M (2005) LabVIEW based data acquisition and processing software programming skills. Microcomputer Information 5:153-154

10. Jiang YH, Sun CJ (2015). Research on pressure fluctuation feature of electric cylinder. Seventh International Conference on Measuring Technology and Mechatronics Automation, Nanchang, China. 893-895

11. He LH, Liu JG, Zhou YS (2009) Error compensation of CVT pressure sensor based on support vector machine. Trans Chinese Soc Agricultural Machinery 40:43-46

12. Zhou H. Design of electric cylinder position servo control system. Dalian Maritime University, 2014

13. Liu ZH, Li Y, Wang ZZ (2012) Investigation of the pressure pulsation control method for load sensitive systems. Hydraul Pneum 6: 40-42

14. Wang G. Research on calibration and measurement system of pressure sensor. Dissertation Sichuan University, 2005 